\title{
Temperature-dependent phonon spectra of magnetic random solid solutions
}

\author{
Yuji Ikeda $\mathbb{D}^{1,2,3}$, Fritz Körmann $\mathbb{D}^{3,4}$, Biswanath Dutta ${ }^{3}{ }^{3}$, Abel Carreras ${ }^{1}$, Atsuto Seko ${ }^{1,2,5,6}$, Jörg Neugebauer ${ }^{3}$ and Isao Tanaka $^{1,2,6,7}$
}

A first-principles-based computational tool for simulating phonons of magnetic random solid solutions including thermal magnetic fluctuations is developed. The method takes fluctuations of force constants due to magnetic excitations as well as due to chemical disorder into account. The developed approach correctly predicts the experimentally observed unusual phonon hardening of a transverse acoustic mode in Fe-Pd an Fe-Pt Invar alloys with increasing temperature. This peculiar behavior, which cannot be explained within a conventional harmonic picture, turns out to be a consequence of thermal magnetic fluctuations. The proposed methodology can be straightforwardly applied to a wide range of materials to reveal new insights into physical behaviors and to design materials through computation, which were not accessible so far.

npj Computational Materials (2018)4:7 ; doi:10.1038/s41524-018-0063-1

\section{INTRODUCTION}

Magnetic random solid solutions represent a large and important class of crystalline materials ranging from structural materials such as steels, ${ }^{1-4}$ including Invar alloys, ${ }^{5-9}$ up to multicomponent magnetic high-entropy alloys. ${ }^{10-12}$ The simultaneous presence of chemical disorder and thermal magnetic fluctuations as well as their couplings to lattice vibrations play pivotal roles in many of these alloys. Lattice vibrations largely dominate thermodynamic properties of materials ${ }^{13}$ and contribute to phase stability, ${ }^{14}$ which is a key parameter for the computational design of new and innovative materials. A computational scheme that simulates the lattice vibrations in magnetic random solid solutions by properly taking into account both, magnetic fluctuations as well as chemical disorder (as sketched in Fig. 1), is therefore of genuine importance.

The delicate interactions between lattice vibrations, chemical disorder, and thermal magnetic fluctuations can cause extreme and unusual physical properties. A prominent example is the hardening of a transverse acoustic phonon mode and elastic constants with increasing temperature in Invar alloys. ${ }^{15,16}$ Since thermal expansion-usually dominating the temperature dependence of phonon modes-is in such alloys negligible, ${ }^{5-9}$ the inclusion of explicit temperature-dependent excitations, such as magnetic fluctuations, is critical to resolve such peculiarities.

In the last few years significant progress has been made for the computation of lattice vibrations incorporating thermal magnetic fluctuations for pure elements such as $\mathrm{Fe}^{17-24}$ and $\mathrm{Ni}^{25}$ as well as for several ordered magnetic compounds. ${ }^{26-30}$ While these methods clearly highlight the recent progress in first-principlesbased thermodynamic approaches for magnetic systems, they are still limited to chemically ordered systems. For random solid solutions without thermal magnetic fluctuations, computational methods for lattice vibrations have also been advanced significantly in the last years. Among such methods, the itinerant coherent-potential approximation (ICPA) $)^{31,32}$ and the band unfolding $^{33-36}$ have been used to successfully compute lattice vibrations for random solid solutions. ${ }^{36-46}$ However, a computational method for lattice vibrations considering both, thermal magnetic fluctuations and chemical disorder, is lacking so far.

We therefore propose a first-principles-based method to calculate lattice vibrations of magnetic random solid solutions, which addresses both, thermal magnetic fluctuations as well as chemical disorder simultaneously. This is achieved by extending and combining the above-mentioned disjunct approaches into a methodological framework, which allows to predict the temperature-dependent phonon spectra of magnetic random solid solutions. To achieve this goal, we apply a two-step procedure and adiabatically decouple the (fast) magnetic and the (slow) chemical degrees of freedom. To include thermal magnetic fluctuations, we utilize force constants (FCs) which implicitly depend on the temperature-dependent magnetic state. $^{22}$ These FCs are obtained using the spin-space averaging method ${ }^{21}$ in combination with quantum Monte Carlo simulations for an effective Heisenberg spin Hamiltonian. To account for chemical disorder, which induces variations of atomic masses and FCs among atomic sites, we employ the ICPA ${ }^{31,32}$ and the band unfolding. ${ }^{33-36}$

\section{RESULTS}

We first calculate the FC s for the two extreme magnetic states: (i) the low-temperature ideal ferromagnetic (FM) state, where all magnetic moments point to the same direction, and (ii) the hightemperature ideal paramagnetic (PM) state, where the magnetic

\footnotetext{
${ }^{1}$ Department of Materials Science and Engineering, Kyoto University, Kyoto 606-8501, Japan; ${ }^{2}$ Center for Elements Strategy Initiative for Structural Materials (ESISM), Kyoto University, Kyoto 606-8501, Japan; ${ }^{3}$ Max-Planck-Institut für Eisenforschung GmbH, D-40237 Düsseldorf, Germany; ${ }^{4}$ Department of Materials Science and Engineering, Delft University of Technology, Mekelweg 2, 2628 CD Delft, Netherlands; ${ }^{5}$ Precursory Research for Embryonic Science and Technology (PRESTO), Japan Science and Technology Agency (JST), Kawaguchi 332-0012, Japan; ${ }^{6}$ Center for Materials Research by Information Integration, National Institute for Materials Science (NIMS), Tsukuba 305-0047, Japan and ${ }^{7}$ Nanostructures Research Laboratory, Japan Fine Ceramics Center, Nagoya 456-8587, Japan Correspondence: Yuji Ikeda (ikeda.yuji.6m@kyoto-u.ac.jp)
}

Received: 28 August 2017 Revised: 2 February 2018 Accepted: 5 February 2018 Published online: 28 February 2018 

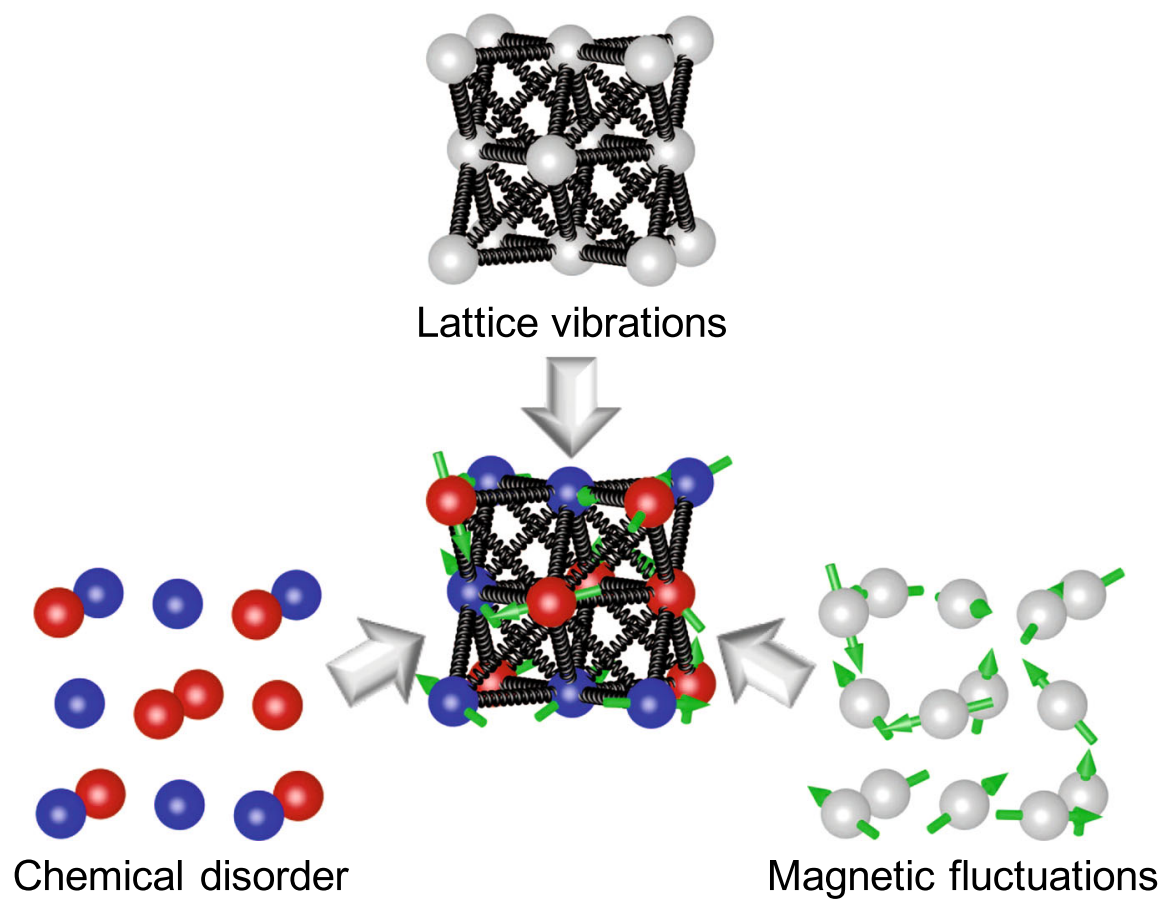

Fig. 1 Sketch of the interplay between lattice vibrations, chemical disorder, and thermal magnetic fluctuations in a crystalline system. Spheres represent atoms, and their colors correspond to different chemical elements. Coils connecting the spheres sketch lattice vibrations, and green arrows represent magnetic moments on atoms. All the three factors have to be simultaneously taken into account to simulate lattice vibrations of magnetic random solid solutions at finite temperature

moments are fully disordered. The FCs in the ideal PM state are obtained using the spin-space averaging method, ${ }^{21,28}$ which consists in a statistical average over a large set of randomly distributed collinear magnetic moments (see Sec. B in the Supplemental Material for further details). The FCs in the magnetic state at temperature $T$, which has magnetic short-range order, are then calculated considering the impact of the magnons as ${ }^{22}$

$$
\Phi_{M-M^{\prime}}(T)=a(T) \Phi_{M-M^{\prime}}^{\mathrm{FM}}+[1-a(T)] \Phi_{M-M^{\prime}}^{\mathrm{PM}},
$$

where $\Phi_{M-M^{\prime}}(T)$ denotes the FCs between the pair of chemical elements $M$ and $M^{\prime}$ at $T$, and $\Phi_{M-M^{\prime}}^{F M}$ and $\Phi_{M-M^{\prime}}^{P M}$ denote the FCs in the ideal FM and in the ideal PM states, respectively. Here, the original formalism for chemically ordered systems ${ }^{22}$ is extended to random solid solutions by considering the element-resolved FCs. The mixing parameter $a(T)$ is directly related to the magnetic energy $^{22}$ (see Sec. $C$ in the Supplemental Material). Spin quantization effects for the magnetic energy and hence for $a(T)$, being critical below $T_{C_{1}}^{47}$ are incorporated by performing numerically precise quantum Monte Carlo simulations for an effective nearest-neighbor Heisenberg spin Hamiltonian. ${ }^{22,47-49}$ Here the solid solution is modeled by randomly distributing the chemical elements having different spin values onto the magnetic sites. Note that the reverse impact of vibrations on the spin Hamiltonian, and hence on the magnetic energetics and $a(T)$, is considered to be small and, therefore, not included in this approach. It is also worth emphasizing that classical Monte Carlo simulations, which do not include spin quantization effects, would result in inaccurate magnetic energetics below $T_{C}{ }^{47}$ and hence inaccurate $a(T)$.

The obtained $\Phi_{M-M^{\prime}}(T)$ are next employed to derive the phonon spectrum of the magnetic random solid solution at any given $T$. In principle both the ICPA ${ }^{31,32}$ and the band unfolding ${ }^{33-36}$ can address the variations of atomic masses and FCs among atomic sites due to chemical disorder. The ICPA is a Green's-functionbased method and analytically incorporates the variations of atomic masses and FCs; a fully random solid solution is rigorously modeled in the ICPA. This method, however, requires the numerical solution of a relatively complex set of equations, ${ }^{31,32}$ making its extension to multicomponent systems challenging. In contrast, the band unfolding is a supercell-based method and can therefore be straightforwardly extended to the multicomponent systems. This method suffers, however, from the undesired periodicity originating from the limited size of the supercell, which may cause spurious features in the computed phonon spectra. To eliminate them, a much larger effective supercell is constructed, and the FCs $\Phi_{M-M^{\prime}}(T)$ computed from the original (smaller) supercell are assigned to the atomic sites of the effective supercell. Such an effective supercell has longer periodicity than the original supercell and therefore includes a larger number of distinct local configurations of chemical elements.

We apply the developed approach to two experimentally wellstudied magnetic alloys, namely to disordered face-centered cubic (fcc) $\mathrm{Fe}_{0.72} \mathrm{Pd}_{0.28}$ and $\mathrm{Fe}_{0.72} \mathrm{Pt}_{0.28}$ alloys. Both Invar alloys reveal the aforementioned characteristic phonon hardening of a $\langle 110\rangle$ (in fractional coordinates for the conventional fcc unit cell) transverse acoustic mode when heated up above the Curie temperature, $T_{C}{ }^{15,50}$ In the following we focus on (i) the impact of mass and FC variations among atomic sites as well as the performance of the ICPA and the band unfolding to incorporate it and, in particular, (ii) the impact of thermal magnetic fluctuations on the phonon spectra.

In order to discuss the impact of mass and FC variations among atomic sites separately from the impact of thermal magnetic fluctuations, we first focus on the ideal FM state $[a(T=0)=1]$, i.e., in the absence of thermal magnetic fluctuations. Figure 2 shows the phonon spectra of $\mathrm{Fe}_{0.72} \mathrm{Pd}_{0.28}$ and $\mathrm{Fe}_{0.72} \mathrm{Pt}_{0.28}$ in the ideal $\mathrm{FM}$ state calculated using the band unfolding (top panels) and the ICPA (middle panels). The results obtained from the two different methods are very similar to each other for both alloys, although the formalisms of the ICPA and of the band unfolding are different as detailed above. Both the methods probably incorporate the impact of chemical disorder into the calculations of phonon spectra in a similar quality. The peak positions of the computed 
(a)
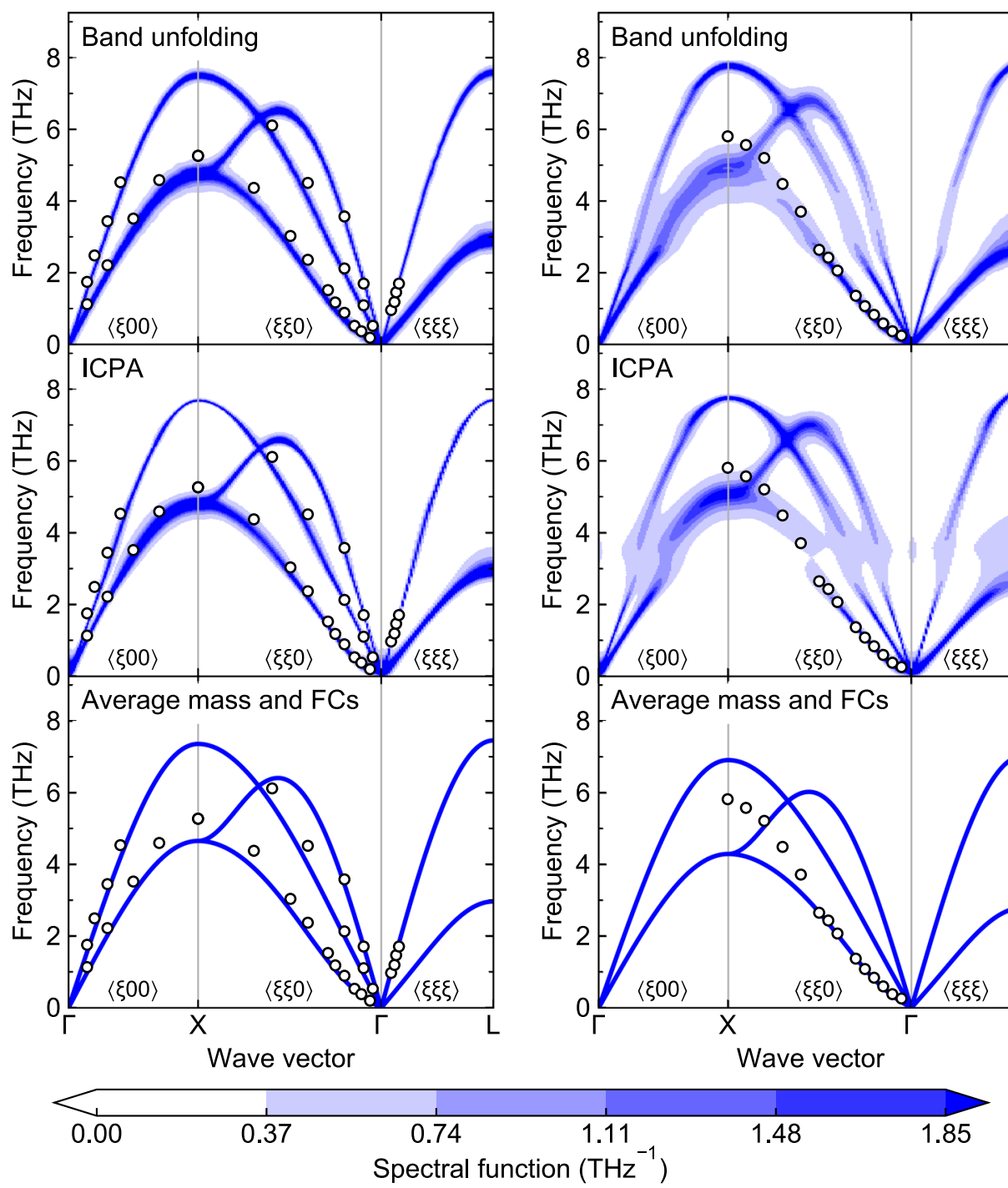

(b)
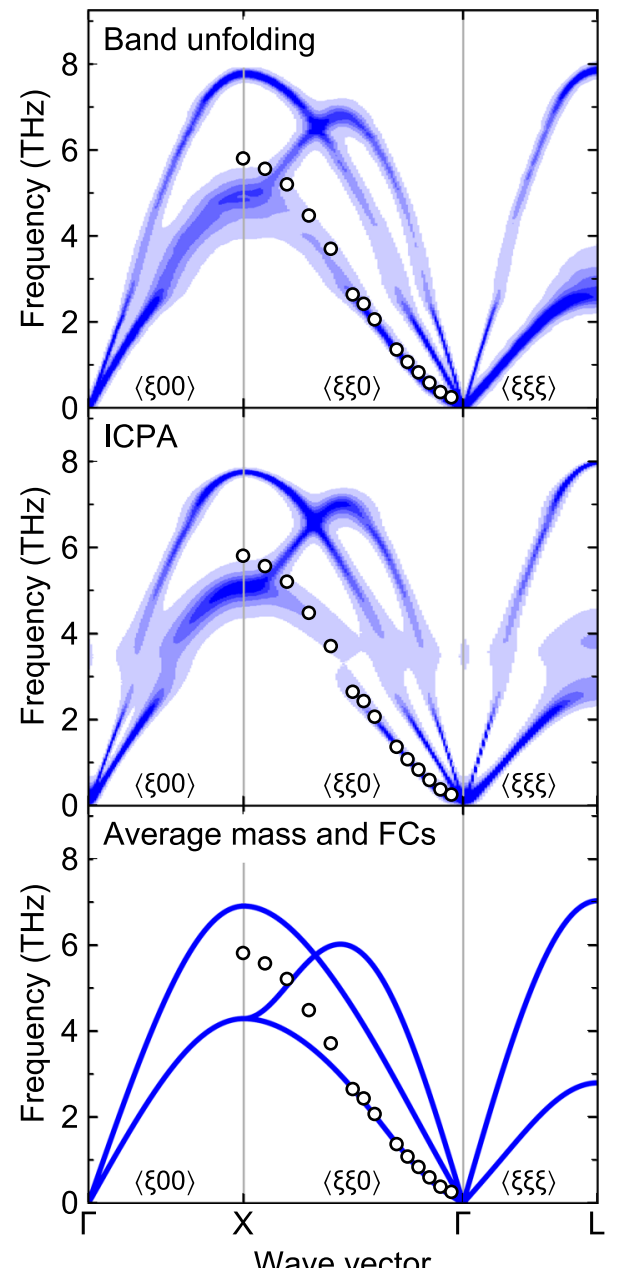

Calc. in the ideal FM

Fig. 2 Phonon spectra of chemically disordered fcc a Fe ${ }_{0.72} \mathrm{Pd}_{0.28}$ and $\mathbf{b} \mathrm{Fe}_{0.72} \mathrm{Pt}_{0.28}$ in the ideal FM state calculated using the band unfolding (top panels) and the ICPA (middle panels). Blue filled contour lines correspond to the magnitude of the spectral functions. Bottom panels show the phonon dispersion relations calculated using the average atomic masses and FCs. White circles show the experimental phonon frequencies of disordered fcc $\mathrm{Fe}_{0.72} \mathrm{Pd}_{0.28}$ at room temperature ${ }^{15}$ and of $\mathrm{Fe}_{0.72} \mathrm{Pt}_{0.28}$ at $12 \mathrm{~K}^{16}$ At these temperatures both disordered alloys are in the FM phase

phonon spectra are also found to be in good agreement with experimental phonon frequencies.

The spectra also show phonon broadening originating from the variations of atomic masses and FCs among atomic sites due to the chemical disorder. For $\mathrm{Fe}_{0.72} \mathrm{Pd}_{0.28}$ this effect is relatively small, while for $\mathrm{Fe}_{0.72} \mathrm{Pt}_{0.28}$ large phonon broadening particularly in the frequency region around $3-5 \mathrm{THz}$ is observed. The large phonon broadening in $\mathrm{Fe}_{0.72} \mathrm{Pt}_{0.28}$ occurs probably due to the large differences of atomic masses and FCs among the chemical elements. The atomic mass of $\mathrm{Pt}$ relative to $\mathrm{Fe}(\approx 3.5)$ is much larger than that of $\mathrm{Pd}(\approx 1.9)$. The $\mathrm{FCs}$ in $\mathrm{Fe}_{0.72} \mathrm{Pt}_{0.28}$ are also largely different among the distinct combinations of the chemical elements compared with those in $\mathrm{Fe}_{0.72} \mathrm{Pd}_{0.28}$ (see Sec. $\mathrm{E}$ in the Supplemental Material).

To elucidate the impacts of the variations of atomic masses and FCs among atomic sites more clearly, the phonon dispersion relations are also calculated in the absence of FC and mass fluctuations using the concentration-weighted average atomic mass $\bar{m}$ and the crystallographically-symmetrized FCs $\bar{\Phi}$. The FCs $\bar{\Phi}$ are obtained by first applying each symmetry operation of the fcc structure to the original FCs and then taking the average of the transformed FCs irrespective of the chemical elements. The results are shown in the bottom panels in Fig. 2. Note that by construction, no phonon broadening is obtained in this case. For the Fe-Pd alloy, the phonon frequencies derived from $\bar{m}$ and $\bar{\Phi}$ still agree reasonably well with those in experiments. For the Fe-Pt alloy, however, the deviations are significant, in particular around the $X$ point. This indicates the importance of taking the variations of atomic masses and FCs among atomic sites into account for accurate phonon computations of random solid solutions. 
Having verified the importance of an appropriate treatment of chemical disorder in random solid solutions, we next analyze the impact of thermal magnetic fluctuations on the phonon spectra from the viewpoint of their temperature dependence. The temperature-dependent spectra are obtained using $\Phi_{M-M^{\prime}}(T)$ from Eq. (1). Calculations are carried out at three representative characteristic temperatures: below, near, and above $T_{C}(575 \mathrm{~K}$ for $\mathrm{Fe}_{0.72} \mathrm{Pd}_{0.28}{ }^{15}$ and $367 \mathrm{~K}$ for $\mathrm{Fe}_{0.72} \mathrm{Pt}_{0.28}{ }^{50}$ ). We focus in particular on the lowest-frequency phonon branch along the $\langle 110\rangle$ direction, which shows the unusual hardening with increasing temperature. Note that within the harmonic approximation and in absence of explicit temperature-dependent excitations, phonon spectra only implicitly depend on temperature via the volume expansion. However, as mentioned above, the considered Invar alloys reveal negligible expansion below $T_{C}$. A conventional harmonic approximation would therefore predict a temperature-independent phonon spectra and cannot explain the experimental data.

Figure 3 shows the results employing the band unfolding (Note that similarly to the discussed ideal FM state (Fig. 2), the ICPA results are again in excellent agreement with the band-unfolding results for the intermediate temperatures and therefore shown only in the Supplemental Material.) For comparison, the results for the ideal FM and the ideal PM states are also shown. The peak positions of the computed temperature-dependent phonon spectra are in agreement with experimental phonon frequencies at the corresponding temperatures, while the results for the ideal FM and the ideal PM states provide the lower and the upper bounds, respectively. This indicates that thermal magnetic fluctuations are crucial to reproduce the experimental data.

Our simulations further indicate the presence of considerable magnetic short-range order (i.e., a finite value of $a(T)$ in Fig. 3) even above $T_{C}$, similar to the one observed for pure Fe. ${ }^{22}$ Without taking magnetic short-range order into account, i.e., by limiting the simulations solely to the ideal PM state, the agreement with experiments is lost. Magnetic short-range order hence plays a crucial role, not only for pure Fe, but also for Fe-based alloys. We also observe slight deviations between our simulations and the experiment above $T_{C}$. Since the Invar effect is lost at the temperature above $T_{C}$, these deviations may be attributed to the increase of the lattice constants, which is not yet included in our calculations.

The temperature dependence of the phonon modes may be also related to the martensitic transformation in these alloys. As observed in Fig. 3, the slope of the lowest-frequency branch along the $\langle 110\rangle$ direction around the $\Gamma$ point largely depends on temperature for both $\mathrm{Fe}_{0.72} \mathrm{Pd}_{0.28}$ and $\mathrm{Fe}_{0.72} \mathrm{Pt}_{0.28}$ alloys and decreases with decreasing temperature. The slope is more than four times smaller in the ideal FM state than in the ideal PM state for both the alloys. Since the slope of this branch is proportional to (a)

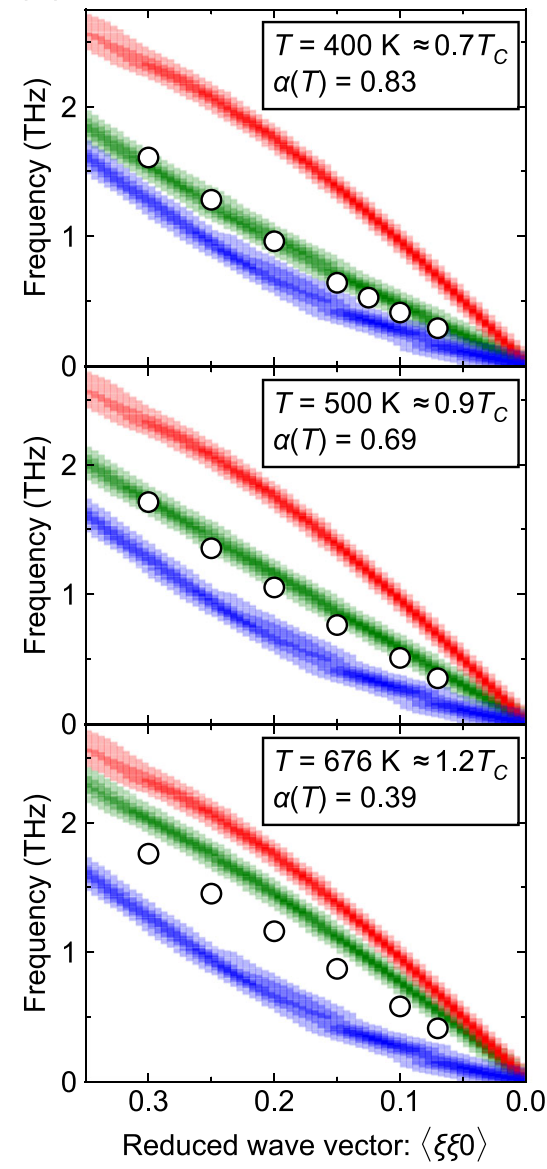

(b)

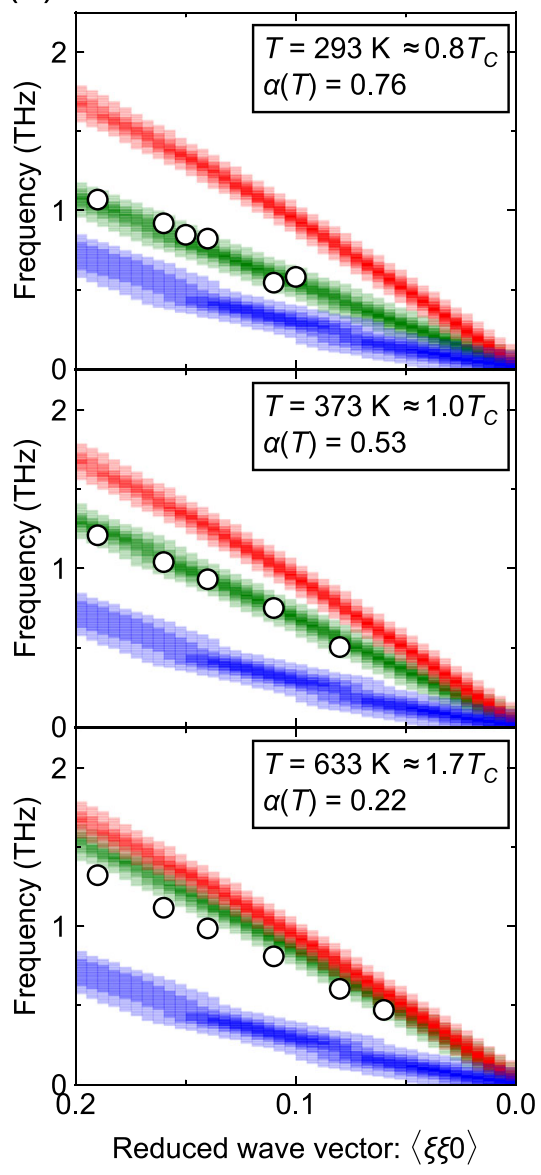

Exp. Calc. in the ideal FM

Calc. in the ideal PM

Calc. at $T$

Fig. 3 Temperature dependence of the phonon spectra for the lowest-frequency branch along the $\langle 110\rangle$ direction (in fractional coordinates for the conventional fcc unit cell) calculated using the band unfolding. a $\mathrm{Fe}_{0.72} \mathrm{Pd}_{0.28}$. b $\mathrm{Fe}_{0.72} \mathrm{Pt}_{0.28}$. Blue, red, and green filled contour lines show the results obtained from the FCs in the ideal FM, in the ideal PM, and at the corresponding temperatures obtained from Eq. (1), respectively. $a(T)$ used to obtain the $\mathrm{FCs}$ at the corresponding temperatures are also given in the panels. White circles show the experimental phonon frequencies for $\mathrm{Fe}_{0.72} \mathrm{Pd}_{0.28}{ }^{15}$ and $\mathrm{Fe}_{0.72} \mathrm{Pt}_{0.28}{ }^{62}$ 
$\sqrt{C^{\prime}},{ }^{51}$ where $C^{\prime}$ is the elastic stiffness constant defined as $C^{\prime} \equiv$ $\left(C_{11}-C_{12}\right) / 2$, it is actually found that $C^{\prime}$ changes dramatically due to the magnetic ordering. $C^{\prime}$ is related to the dynamical stability along the Bain path, ${ }^{52}$ and therefore the softening of the $\langle 110\rangle$ phonon branch is commonly considered as a precursor of the martensitic transformation. In experiments, temperature-induced martensitic transformations are indeed observed in disordered fcc $\mathrm{Fe}_{1-x} \mathrm{Pd}_{x}$ and $\mathrm{Fe}_{1-x} \mathrm{Pt}_{x}(x=0.25-0.33)$ alloys, e.g., at $314.5 \mathrm{~K}^{53}$ for $\mathrm{Fe}_{0.72} \mathrm{Pd}_{0.28}$ and approximately $125 \mathrm{~K}^{54}$ for $\mathrm{Fe}_{0.73} \mathrm{Pt}_{0.27}$. Having identified magnetic fluctuations as a driving force for the softening of the $\langle 110\rangle$ phonon branch, our results suggest that the martensitic transformation in these Fe-based alloys is driven by magnon-phonon contributions.

Finally, we show the supercell-size dependence of the phonon spectra calculated using the band unfolding approach. We considered three supercell models with different sizes, namely the 32 -atom $2 \times 2 \times 2$, the 256 -atom $4 \times 4 \times 4$, and the 846 -atom $6 \times 6 \times 6$ supercells of the conventional fcc unit cell. The atomic configurations of the $2 \times 2 \times 2$ supercell models were the same as those of the special-quasirandom-structure $(S Q S)^{55}$ models used to extract the FCs, while the atomic configurations of the larger supercell models were determined using a pseudorandom number generator. Figure 4 shows the unfolded phonon spectra of the $\mathrm{Fe}_{0.72} \mathrm{Pd}_{0.28}$ and $\mathrm{Fe}_{0.72} \mathrm{Pt}_{0.28}$ in the ideal $\mathrm{FM}$ state obtained from these supercell models. Even for the smallest $2 \times 2 \times$ 2 supercell models (top panels), the phonon spectra are qualitatively similar to those obtained from the larger supercell models. The spectra obtained from the $2 \times 2 \times 2$ supercell models are, however, less smooth than those obtained from the larger supercell models. The ruggedness in the spectra obtained from the $2 \times 2 \times 2$ supercell models is due to the small number of local environments sampled around the atoms. In contrast, the spectra obtained from the $4 \times 4 \times 4$ supercell models (middle panels) and from the $6 \times 6 \times 6$ supercell models (bottom panels) are smooth and very similar to each other for both alloys. This indicates that the spectra obtained from the $6 \times 6 \times 6$ supercell models are almost converged with respect to the supercell size. (a)

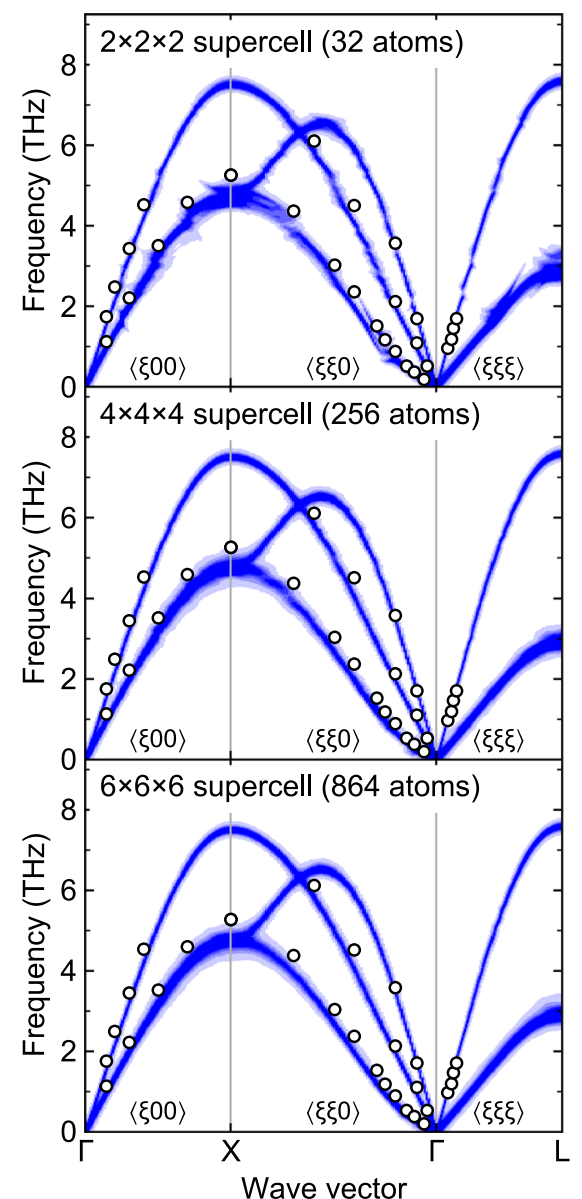

(b)

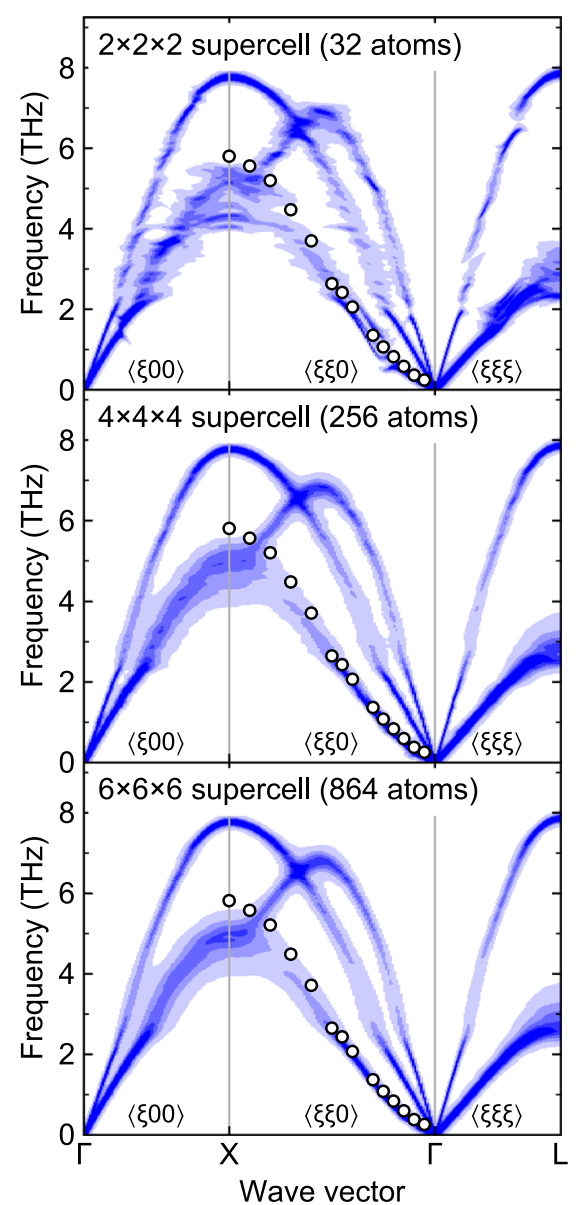

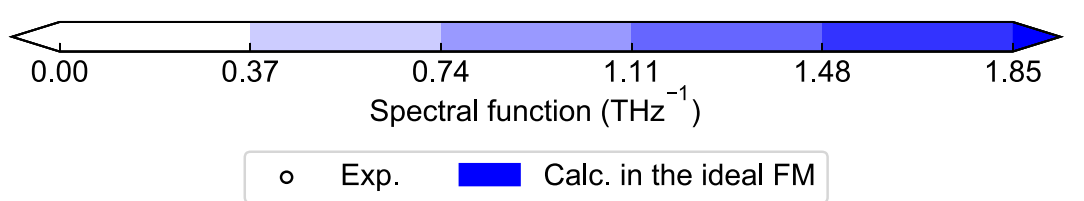

Fig. 4 Unfolded phonon spectra of a $\mathrm{Fe}_{0.72} \mathrm{Pd}_{0.28}$ and $\mathbf{b} \mathrm{Fe}_{0.72} \mathrm{Pt}_{0.28}$ in the ideal $\mathrm{FM}$ state obtained from the supercell models with three different sizes. Blue filled contour lines correspond to the relative magnitude of the spectral functions. White circles show the experimental phonon frequencies for disordered fcc $\mathrm{Fe}_{0.72} \mathrm{Pd}_{0.28}$ at room temperature ${ }^{15}$ and $\mathrm{Fe}_{0.72} \mathrm{Pt}_{0.28}$ at $12 \mathrm{~K}^{16}$ At these temperatures both these disordered alloys are in the FM phase 


\section{DISCUSSION}

We propose a first-principles-based method to incorporate both thermal magnetic fluctuations and chemical disorder into a unified computational framework of phonon calculations for magnetic random solid solutions. Chemical disorder, which leads to variations of atomic masses and FCs among atomic sites, is taken into account using the ICPA and the band unfolding in combination with SQSs. Thermal magnetic fluctuations are incorporated using the spin-space averaging and quantum Monte Carlo simulations using an effective Heisenberg spin Hamiltonian.

The proposed methodology is applied to Fe-Pd and Fe-Pt Invar alloys. Both the ICPA and the band unfolding are found to be equally capable for computing phonon spectra of the chemically disordered alloys. Both methods also reveal phonon broadening due to the chemical disorder. Taking thermal magnetic fluctuations into account, the developed approach shows excellent agreement between the peak positions of the computed temperature-dependent phonon spectra and experimental phonon frequencies. In particular, the approach correctly reproduces the experimentally observed unusual hardening in iron-based Invar alloys at high temperatures and shows that magnetic fluctuations are responsible for it. Finite-temperature magnon-phonon contributions also cause the experimentally observed softening of the elastic stiffness constant $C^{\prime}$ and hence trigger the martensitic transformation in these alloys.

The proposed approach can be straightforwardly applied to other complex magnetic random solid solutions such as magnetic high-entropy alloys, which are expected to reveal complex physical mechanisms caused by the interplay between thermal magnetic fluctuations, chemical disorder, and lattice vibrations. It may be also possible to apply the proposed approach to magnetic random solid solutions with chemical short-range order; e.g., a supercell model of a disordered alloy with chemical short-range order can be used for the band unfolding.

\section{METHODS}

Chemical and magnetic (to obtain the FCs in the ideal PM state) disorder in these alloys were simulated by $\mathrm{SQSs}^{55}$ based on the 32 -atom $2 \times 2 \times$ 2 supercell of the conventional fcc unit cell (see Sec. A in the Supplemental Material). Chemical compositions for the SQS supercell models were chosen to be $\mathrm{Fe}_{0.75} \mathrm{Pd}_{0.25}$ and $\mathrm{Fe}_{0.75} \mathrm{Pt}_{0.25}$, being close to the experimental ones, namely, $\mathrm{Fe}_{0.72} \mathrm{Pd}_{0.28}$ and $\mathrm{Fe}_{0.72} \mathrm{Pt}_{0.28}$, respectively.

The mixing parameter $a(T)$ in Eq. (1) was determined using an effective Heisenberg spin Hamiltonian for a $7 \times 7 \times 7$ supercell including 1372 magnetic sites (see Sec. C in the Supplemental Material). The FCs of the SQS models were calculated using finite displacements of $0.01 \AA$. The FCs in the ideal PM state, $\Phi_{M-M^{\prime}}^{P M}$, were obtained using the spin-space averaging method $^{21}$ by applying all inequivalent symmetry operations of the fcc structure to the SQS models with a disordered magnetic configuration (See also Sec. B in the Supplemental Material). Applying the symmetry operations to the SQS disordered-magnetic configuration, multiple different disordered magnetic configurations are obtained. At the magnetic high-temperature limit, such disordered magnetic configurations are dominant and have equal weights. ${ }^{21,22}$ The averaging of the FCs was performed for each chemically inequivalent pair in the disordered-alloy model (e.g., Fe-Fe, Fe-Pd, Pd-Pd). For the chosen SQS model there are 1536 (=48 rotations $\times 32$ translations) inequivalent symmetry operations. To be specific, taking the first nearest neighbor FCs of the Fe-Fe pair in the ideal PM state as an example, the statistical average with equal weights was computed over the FCs of $864(=1536 \times 0.75 \times 0.75)$ atomic pairs in the SQS models. The FCs up to the fourth nearest neighbors were then employed in the ICPA and the band unfolding. For the band-unfolding procedure, the obtained $\Phi_{M-M^{\prime}}(T)$ were assigned to the atomic sites of effective 864-atom $6 \times 6 \times 6$ supercell models, where the composition ratios were fixed to $\mathrm{Fe}_{0.75} \mathrm{Pd}_{0.25}$ and $\mathrm{Fe}_{0.75} \mathrm{Pt}_{0.25}$ to be close to the experimental ones.

Electronic structures were calculated in the framework of densityfunctional theory within the generalized gradient approximation of the Perdew-Burke-Ernzerhof from $^{56}$ using the plane-wave basis projector augmented-wave method $^{57}$ as implemented in the vasp code. ${ }^{58-60}$
A plane-wave energy cutoff of $350 \mathrm{eV}$ was used. Since thermal expansion is small for the chosen Invar alloys, fixed lattice constants were applied for the computer simulations, which were taken from experimental data at room temperature; $3.755 \AA^{15}$ (disordered fcc $\mathrm{Fe}_{0.72} \mathrm{Pd}_{0.28}$ ) and $3.749 \AA^{61}$ (disordered fcc $\mathrm{Fe}_{0.72} \mathrm{Pt}_{0.28}$ ). Internal atomic positions were fully relaxed while keeping the lattices of the SQS models fixed.

\section{Data availability}

The authors declare that all the data supporting the findings of this study are available within the paper and its Supplemental Material.

\section{ACKNOWLEDGEMENTS}

Funding by the Ministry of Education, Culture, Sports, Science, and Technology (MEXT); Japan, through Elements Strategy Initiative for Structural Materials (ESISM) of Kyoto University; by the Japan Society for the Promotion of Science (JSPS) KAKENHI Grant-in-Aid for Young Scientist (B) (Grant No. 16K18228); by the European Research Council under the EU's 7th Framework Programme (FP7/2007-2013)/ERC Grant agreement 290998; the Grant-in-Aid for Scientific Research on Innovative Areas Nano Informatics (Grant No. 25106005) from the Japan Society for the Promotion of Science (JSPS); by the Deutsche Forschungsgemeinschaft (DFG) for the scholarship KO 5080/1-1; and by the DFG for their funding within the priority programme SPP 1599. All funding is gratefully acknowledged.

\section{AUTHOR CONTRIBUTIONS}

All authors designed the project, discussed the results, and wrote the manuscript. Y.I. performed the electronic-structure calculations and applied the band unfolding, which was supported by A.C., A.S., and I.T. F.K. and B.D. performed the quantum Monte Carlo simulations and the ICPA calculations, respectively, which were supported by J.N.

\section{ADDITIONAL INFORMATION}

Supplementary information accompanies the paper on the npj Computational Materials website (https://doi.org/10.1038/s41524-018-0063-1).

Competing interests: The authors declare no competing financial interests.

Publisher's note: Springer Nature remains neutral with regard to jurisdictional claims in published maps and institutional affiliations.

\section{REFERENCES}

1. Muñoz, J. A. et al. Positive vibrational entropy of chemical ordering in FeV. Phys. Rev. Lett. 107, 115501 (2011).

2. Razumovskiy, V. I., Ruban, A. V. \& Korzhavyi, P. A. Effect of temperature on the elastic anisotropy of pure $\mathrm{Fe}$ and $\mathrm{Fe}_{0.9} \mathrm{Cr}_{0.1}$ random alloy. Phys. Rev. Lett. 107, 205504 (2011).

3. Vitos, L., Korzhavyi, P. A. \& Johansson, B. Elastic property maps of austenitic stainless steels. Phys. Rev. Lett. 88, 155501 (2002).

4. Vitos, L., Korzhavyi, P. A. \& Johansson, B. Stainless steel optimization from quantum mechanical calculations. Nat. Mater. 2, 25-28 (2003).

5. Weiss, R. J. The origin of the 'invar' effect. Proc. Phys. Soc. 82, 281-288 (1963).

6. van Schilfgaarde, M., Abrikosov, I. A. \& Johansson, B. Origin of the Invar effect in iron-nickel alloys. Nature 400, 46-49 (1999).

7. Khmelevskyi, S., Turek, I. \& Mohn, P. Large negative magnetic contribution to the thermal expansion in iron-platinum alloys: quantitative theory of the Invar effect. Phys. Rev. Lett. 91, 037201 (2003).

8. Yokoyama, T. \& Eguchi, K. Anharmonicity and quantum effects in thermal expansion of an Invar alloy. Phys. Rev. Lett. 107, 065901 (2011).

9. Yokoyama, T. \& Eguchi, K. Anisotropic thermal expansion and cooperative Invar and anti-Invar effects in Mn alloys. Phys. Rev. Lett. 110, 075901 (2013).

10. Zhang, Y. et al. Influence of chemical disorder on energy dissipation and defect evolution in concentrated solid solution alloys. Nat. Commun. 6, 8736 (2015).

11. Granberg, F. et al. Mechanism of radiation damage reduction in equiatomic multicomponent single phase alloys. Phys. Rev. Lett. 116, 135504 (2016).

12. Li, Z., Pradeep, K. G., Deng, Y., Raabe, D. \& Tasan, C. C. Metastable high-entropy dualphase alloys overcome the strength-ductility trade-off. Nature 534, 227-230 (2016).

13. Fultz, B. Vibrational thermodynamics of materials. Prog. Mater. Sci. 55, 247-352 (2010).

14. Fultz, B. Phase Transitions in Materials. (Cambridge University Press, Cambridge, 2014). 
15. Sato, M., Grier, B. H., Shapiro, S. M. \& Miyajima, H. Effect of magnetic ordering on the lattice dynamics of fcc $\mathrm{Fe}_{1-x} \mathrm{Pd}_{x}$. J. Phys. F. 12, 2117 (1982).

16. Kästner, J. et al. Influence of atomic order on $\mathrm{TA}_{1}[110]$ phonon softening and displacive phase transition in $\mathrm{Fe}_{72} \mathrm{Pt}_{28}$ invar alloys. Eur. Phys. J. B 10, 641-648 (1999).

17. Leonov, I., Poteryaev, A. I., Anisimov, V. I. \& Vollhardt, D. Calculated phonon spectra of paramagnetic iron at the $a-\gamma$ phase transition. Phys. Rev. B 85, 020401 (2012).

18. Leonov, I. et al. Electronic correlations determine the phase stability of iron up to the melting temperature. Sci. Rep. 4, 5585 (2014).

19. Ruban, A. V. \& Razumovskiy, V. I. Spin-wave method for the total energy of paramagnetic state. Phys. Rev. B 85, 174407 (2012).

20. Ruban, A. V., Razumovskiy, V. I. \& Körmann, F. Erratum: spin-wave method for the total energy of paramagnetic state [Phys. Rev. B 85, 174407 (2012)]. Phys. Rev. B 89, 179901(E) (2014).

21. Körmann, F., Dick, A., Grabowski, B., Hickel, T. \& Neugebauer, J. Atomic forces at finite magnetic temperatures: phonons in paramagnetic iron. Phys. Rev. $B \mathbf{8 5}$ 125104 (2012).

22. Körmann, F. et al. Temperature dependent magnon-phonon coupling in bcc Fe from theory and experiment. Phys. Rev. Lett. 113, 165503 (2014).

23. Ikeda, Y., Seko, A., Togo, A. \& Tanaka, I. Phonon softening in paramagnetic bcc Fe and its relationship to the pressure-induced phase transition. Phys. Rev. B 90 134106 (2014).

24. Alling, B. et al. Strong impact of lattice vibrations on electronic and magnetic properties of paramagnetic fe revealed by disordered local moments molecular dynamics. Phys. Rev. B 93, 224411 (2016).

25. Körmann, F., Ma, P.-W., Dudarev, S. L. \& Neugebauer, J. Impact of magnetic fluctuations on lattice excitations in fcc nickel. J. Phys. Condens. Matter 28, 076002 (2016).

26. Fennie, C. J. \& Rabe, K. M. Magnetically induced phonon anisotropy in $\mathrm{ZnCr}_{2} \mathrm{O}_{4}$ from first principles. Phys. Rev. Lett. 96, 205505 (2006).

27. Shulumba, N. et al. Vibrational free energy and phase stability of paramagnetic and antiferromagnetic CrN from ab initio molecular dynamics. Phys. Rev. B 89, 174108 (2014).

28. Zhou, L. et al. Structural stability and thermodynamics of $\mathrm{CrN}$ magnetic phases from $a b$ initio calculations and experiment. Phys. Rev. B 90, 184102 (2014).

29. Gruner, M. E. et al. Element-resolved thermodynamics of magnetocaloric $\mathrm{LaFe}_{13}$ ${ }_{-} \mathrm{Si}_{\mathrm{x}}$. Phys. Rev. Lett. 114, 057202 (2015)

30. Dutta, B. et al. Ab initio prediction of martensitic and intermartensitic phase boundaries in Ni-Mn-Ga. Phys. Rev. Lett. 116, 025503 (2016).

31. Ghosh, S., Leath, P. L. \& Cohen, M. H. Phonons in random alloys: the itinerant coherent-potential approximation. Phys. Rev. B 66, 214206 (2002).

32. Ghosh, S., Leath, P. L. \& Cohen, M. H. Erratum: phonons in random alloys: the itinerant coherent-potential approximation [Phys. Rev. B 66, 214206 (2002)]. Phys. Rev. B 68, 019903(E) (2003)

33. Boykin, T. B., Kharche, N., Klimeck, G. \& Korkusinski, M. Approximate bandstructures of semiconductor alloys from tight-binding supercell calculations. J. Phys. Condens. Matter 19, 036203 (2007).

34. Allen, P. B., Berlijn, T., Casavant, D. A. \& Soler, J. M. Recovering hidden Bloch character: unfolding electrons, phonons, and slabs. Phys. Rev. B 87, 085322 (2013).

35. Allen, P. B., Berlijn, T., Casavant, D. A. \& Soler, J. M. Erratum: recovering hidden Bloch character: unfolding electrons, phonons, and slabs [Phys. Rev. B 87, 085322 (2013)]. Phys. Rev. B 87, 239904(E) (2013).

36. Ikeda, Y., Carreras, A., Seko, A., Togo, A. \& Tanaka, I. Mode decomposition based on crystallographic symmetry in the band-unfolding method. Phys. Rev. B 95, 024305 (2017).

37. Dutta, B. \& Ghosh, S. The phonon spectra and elastic constants of $\mathrm{Pd}_{x} \mathrm{Fe}_{1-x}$ : an understanding from inter-atomic interactions. J. Phys. Condens. Matter 21, 095411 (2009).

38. Dutta, B. \& Ghosh, S. Phonon spectra of $\mathrm{Pd}_{x} \mathrm{Fe}_{1-x}$ alloys with transferable force constants. J. Phys. Condens. Matter 21, 395401 (2009).

39. Dutta, B., Bisht, K. \& Ghosh, S. Ab initio calculation of phonon dispersions in sizemismatched disordered alloys. Phys. Rev. B 82, 134207 (2010).

40. Dutta, B. \& Ghosh, S. Vibrational properties of $\mathrm{Ni}_{x} \mathrm{Pt}_{1-x}$ alloys: an understanding from ab initio calculations. J. Appl. Phys. 109, 053714 (2011).

41. Grånäs, O., Dutta, B., Ghosh, S. \& Sanyal, B. A new first principles approach to calculate phonon spectra of disordered alloys. J. Phys. Condens. Matter 24, 015402 (2012)
42. Boykin, T. B., Ajoy, A., llatikhameneh, H., Povolotskyi, M. \& Klimeck, G. Brillouin zone unfolding method for effective phonon spectra. Phys. Rev. B 90, 205214 (2014).

43. Huang, $\mathrm{H}$. Effect of extended line defects on thermal conduction of carbon nanotubes: analyzing phonon structures by band unfolding. J. Phys. Condens. Matter 27, 305402 (2015).

44. Zheng, F. \& Zhang, P. Phonon dispersion unfolding in the presence of heavy breaking of spatial translational symmetry. Comput. Mater. Sci. 125, 218-223 (2016).

45. Overy, A. R., Simonov, A., Chater, P. A., Tucker, M. G. \& Goodwin, A. L. Phonon broadening from supercell lattice dynamics: random and correlated disorder Phys. Status Solidi B 254, 1600586 (2017).

46. Körmann, F., Ikeda, Y., Grabowski, B. \& Sluiter, M. H. F. Phonon broadening in high entropy alloys. npj Comput. Mater. 3, 36 (2017).

47. Körmann, F., Dick, A., Hickel, T. \& Neugebauer, J. Rescaled monte carlo approach for magnetic systems: $A b$ initio thermodynamics of bcc iron. Phys. Rev. B $\mathbf{8 1}$ 134425 (2010).

48. Körmann, F., Dick, A., Hickel, T. \& Neugebauer, J. Role of spin quantization in determining the thermodynamic properties of magnetic transition metals. Phys. Rev. B 83, 165114 (2011).

49. Körmann, F. et al. Thermodynamic modeling of chromium: strong and weak magnetic coupling. J. Phys. Condens. Matter 25, 425401 (2013).

50. Kawald, U., Zemke, W., Bach, H., Pelzl, J. \& Saunders, G. Elastic constants and martensitic phase transitions in FePt and FeNiPt invar alloys. Phys. B 161, 72-74 (1990).

51. Kittel, C. Introduction to Solid State Physics. (Wiley, New York, 2004).

52. Bain, E. C. \& Dunkirk, N. The nature of martensite. Trans. Am. Inst. Min. Metall. Eng. 70, 25 (1924).

53. Arabi-Hashemi, A., Ma, Y., Setzer, A., Esquinazi, P. \& Mayr, S. Shifting martensite transformation temperatures of single crystalline $\mathrm{Fe}_{72} \mathrm{Pd}_{28}$ thin films by external magnetic fields. Scr. Mater. 104, 91-94 (2015).

54. Sumiyama, K., Shiga, M. \& Nakamura, Y. Characteristic martensitic transformation of the Fe-Pt disordered alloys around the $\gamma-a$ phase boundary. Phys. Status Solidi A 76, 747-751 (1983)

55. Zunger, A., Wei, S.-H., Ferreira, L. G. \& Bernard, J. E. Special quasirandom structures. Phys. Rev. Lett. 65, 353-356 (1990).

56. Perdew, J. P., Burke, K. \& Ernzerhof, M. Generalized gradient approximation made simple. Phys. Rev. Lett. 77, 3865-3868 (1996)

57. Blöchl, P. E. Projector augmented-wave method. Phys. Rev. B 50, 17953-17979 (1994).

58. Kresse, G. Ab initio molecular dynamics for liquid metals. J. Non-Cryst. Solids 192 193, 222-229 (1995)

59. Kresse, G. \& Furthmüller, J. Efficiency of ab-initio total energy calculations for metals and semiconductors using a plane-wave basis set. Comput. Mater. Sci. 6 , 15-50 (1996)

60. Kresse, G. \& Joubert, D. From ultrasoft pseudopotentials to the projector augmented-wave method. Phys. Rev. B 59, 1758-1775 (1999).

61. Sumiyama, K., Shiga, M. \& Nakamura, Y. Magnetization, thermal expansion and low temperature specific heat of $\mathrm{Fe}_{72} \mathrm{Pt}_{28}$ Invar alloy. J. Phys. Soc. Jpn. 40, 996-1001 (1976)

62. Endoh, Y. Lattice dynamics in ferromagnetic Invar alloys. J. Magn. Magn. Mater 10, 177-182 (1979)

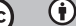

Open Access This article is licensed under a Creative Commons Attribution 4.0 International License, which permits use, sharing, adaptation, distribution and reproduction in any medium or format, as long as you give appropriate credit to the original author(s) and the source, provide a link to the Creative Commons license, and indicate if changes were made. The images or other third party material in this article are included in the article's Creative Commons license, unless indicated otherwise in a credit line to the material. If material is not included in the article's Creative Commons license and your intended use is not permitted by statutory regulation or exceeds the permitted use, you will need to obtain permission directly from the copyright holder. To view a copy of this license, visit http://creativecommons. org/licenses/by/4.0/.

(c) The Author(s) 2018 